\title{
Psychiatric Diagnoses in Patients with Williams Syndrome and Their Families
}

Janet C. Kennedy M.D. (PGY1)

jckennedy@partners.org

David L. Kaye M.D.

Laurie S. Sadler M.D.

Follow this and additional works at: https://jdc.jefferson.edu/jeffjpsychiatry

Part of the Psychiatry Commons

Let us know how access to this document benefits you

\section{Recommended Citation}

Kennedy, Janet C. M.D. (PGY1); Kaye, David L. M.D.; and Sadler, Laurie S. M.D. (2006) "Psychiatric

Diagnoses in Patients with Williams Syndrome and Their Families," Jefferson Journal of Psychiatry. Vol.

20 : Iss. 1 , Article 4.

DOI: https://doi.org/10.29046/JJP.020.1.003

Available at: https://jdc.jefferson.edu/jeffjpsychiatry/vol20/iss1/4

This Article is brought to you for free and open access by the Jefferson Digital Commons. The Jefferson Digital Commons is a service of Thomas Jefferson University's Center for Teaching and Learning (CTL). The Commons is a showcase for Jefferson books and journals, peer-reviewed scholarly publications, unique historical collections from the University archives, and teaching tools. The Jefferson Digital Commons allows researchers and interested readers anywhere in the world to learn about and keep up to date with Jefferson scholarship. This article has been accepted for inclusion in Jefferson Journal of Psychiatry by an authorized administrator of the Jefferson Digital Commons. For more information, please contact: JeffersonDigitalCommons@jefferson.edu. 


\section{Psychiatric Diagnoses in Patients with Williams Syndrome and Their Families}

Janet C. Kennedy, M.D. (PGY1), David L. Kaye, M.D., Laurie S. Sadler, M.D.

\section{ABSTRACT}

Williams Syndrome (WS) is a genetic disorder associated with mental retardation (MR) and a distinct behavioral phenotype including a friendly and outgoing personality. This population, like others with MR, has been reported to have an increased rate of symptoms of mental illness; however, few studies have used DSM-IV criteria to quantify specific psychiatric diagnoses in WS and the prevalence of psychiatric illness in relatives of individuals with WS and the possible relationship between family and patient diagnoses is currently unknown.

Methods: Twenty-one families participated; the patients' average age was 16 years. DSM-IV diagnoses were applied by using the Anxiety Disorders Interview Schedule (ADIS, Parent and Child Versions) and the Family History Screen.

Results: A diagnosis from the ADIS was applicable to 13 patients (62\%), and in 16 patients $(\mathbf{7 6 \% )}$ a diagnosis was applicable in their first-degree family members. Ten patients $(\mathbf{4 8 \%})$ had some form of anxiety, specific phobia being the most common. Three patients $(14 \%)$ had major depressive disorder and 9 patients $(43 \%)$ had attention-deficit/hyperactivity disorder (ADHD). The presence of anxiety or mood disorders in patients with WS and the presence of these disorders in their family members were unrelated.

Conclusions: Patients with WS have a high prevalence of anxiety disorders and of ADHD. The presence of psychiatric disorders in WS did not appear to have a significant relationship to family history of psychiatric disorders, consistent with the hypothesis that the specific genetic alteration in WS causes, or contributes to causing, the anxiety disorders and the ADHD that are so common in the disorder.

Populations with mental retardation (MR), especially those with known genetic alterations, offer a window into the effects of genes on determining cognition and behavior. Populations with MR are known to have increased rates of emotional distress and mental illness $(1,2)$. While estimates vary, the prevalence of 
psychiatric disorders in individuals with MR is reported as three to four times than in the general population (3). Of children with MR living in the community, 20$35 \%$ have been found to have a comorbid mental illness (2). Reported rates of psychiatric diagnoses in this population have varied, ranging from $12 \%$ to $42 \%$ (2). More recent studies demonstrate the prevalence of psychiatric disorders in MR children to be $33-40 \%(4,5)$. When DSM-IV criteria are used, 39\% of a cohort of mentally retarded children have a diagnosable mental illness (6)

Williams syndrome (WS) serves as a prototype for studying the relationship between genetics and behavior because affected patients have a strikingly similar behavioral phenotype. WS results from a deletion that includes the elastin gene on chromosome 7q11.23 and occurs in 1 in 10,000-20,000 births $(7,8)$. Most cases result from a new mutation. The associated mental retardation generally results in an IQ between 41 and 80 (8). Other characteristics of WS include short stature, specific facial features, hyperacusis, and cardiovascular defects. Individuals with WS demonstrate relative strengths in auditory rote memory and verbal ability. Compared to other mentally retarded individuals with similar IQ, children with WS speak more fluently, possess larger vocabularies, and are better conversationalists (8). Additionally, these patients are highly sociable, an affability sometimes described as a "cocktail party personality": talkative, overfriendly, and outgoing; they notably lack stranger anxiety.

Individuals with WS frequently exhibit psychiatric symptoms: generalized anxiety and worries, negative mood, and sensitivity to criticism. Behavioral and emotional disturbances have been reported in up to $80 \%$ of children with WS, much higher than in other mentally retarded populations (9). They have significantly more fears as well as a wider range of frequently occurring fears as compared to other mentally retarded populations, and also have an increased prevalence of anticipatory anxiety (10).

Even so, few studies have used DSM-IV criteria to quantify specific psychiatric diagnoses. Additionally, there is little research examining the possible relationship between family history of psychiatric illness and psychiatric diagnoses in WS patients. This study aims to identify the prevalence and nature of psychiatric disorders in patients with WS using standard DSM-IV criteria. The study also aims to determine the prevalence of psychiatric disorders in first-degree relatives of WS patients and examine the possible relationship between family history of psychiatric illness and patient symptomatology. We hypothesized that if mood, anxiety, or ADHD disorders were a part of the WS behavioral phenotype in and of itself, there would be no relationship between the presence of those disorders in patients and their presence in family members.

Jefferson Journal of Psychiatry, Volume 20, Number 1

ISSN 1935-0783

(C) 2006 by the authors

On the Web: jdc.jefferson.edu/jeffjpsychiatry 


\section{METHODS}

All patients age 7 years or older who have been evaluated at the Williams Syndrome Clinic of Women and Children's Hospital of Buffalo, NY were contacted by mail and telephone to participate in the study. The Williams Syndrome Clinic is a multidisciplinary center that regularly evaluates patients from a large geographic area. Patients were excluded from the study if a biological parent was not the primary caregiver. Twenty-one of 28 eligible families agreed to participate. All were Caucasian. All children with WS lived at home with one or both parents. They ranged in age from 7-28 years (mean 16 years); 14 of the 21 patients were female. All patients had WS that was confirmed by fluorescence in situ hybridization (FISH) analysis. Seven patients had currently or previously taken one or more pharmacologic agents for treatment of psychiatric or behavioral symptoms.

Patients and both parents, when available, were interviewed in their own homes by a single examiner (JCK). Seventeen families (81\%) had both parents present at the time of interview. Subjects and parents received information on the nature of the study and the assessment tools; subjects and the parents that were present gave written informed consent. The study methods were approved by the Institutional Review Board of Women and Children's Hospital of Buffalo. Parent and child responses and diagnoses were reviewed by a child and adolescent psychiatrist (DLK).

\section{Measurements}

The Anxiety Disorders Interview Schedule (ADIS) is a standardized semistructured interview that uses DSM-IV criteria to assess for 14 psychiatric disorders in children and adolescents, including anxiety, specific phobia, MDD, and ADHD (11). The ADIS was administered separately to the parents and the individual with WS (using both Parent and Child Versions) to arrive at a single combined score. In order for a diagnosis to be assigned, symptom-criteria must be met and must interfere with functioning. An impairment in functioning is defined by a minimum score of 4 on a scale of 0 to 8 , as rated by both the subject and the parents in answer to the questions: "How much has [the problem] messed things up for you with friends, in school, or at home? How much does it stop you from doing things you would like to do?”

The Family History Screen (FHS) is a semi-structured interview assessing lifetime history for 15 psychiatric disorders in subjects and first-degree relatives (parents and siblings) (12). The FHS was administered separately to each 
biological parent. Diagnoses queried included anxiety, specific phobia, MDD, and ADHD.

\section{Data Analysis}

Descriptive analyses were used for the prevalence and distribution of psychiatric diagnoses in the study population. The association between patient and family history of selected disorders was analyzed using Fisher's exact tests for contingency tables. Disorders examined in subjects and family members were MDD, any anxiety, specific phobia, and ADHD, as these were expected to be the most prevalent based on previous studies $(1,13)$. Because anxiety and depression may occur together, analyses were also performed for any mood or anxiety disorder. Fisher's exact test was chosen over the Chi-square test for independence because of the small cell- frequencies (expected cell frequencies $\leq 5$ ) in all of the 2x2 contingency tables. For Fisher's exact test, the null hypotheses of independence was performed at a 0.05 significance level. All of the statistical analyses are performed on SAS version-9.

\section{RESULTS}

\section{DSM-IV Diagnoses in WS population}

Thirteen of 21 subjects (62\%) had at least one DSM-IV diagnosis (Table 1). The most common were: any anxiety disorder (48\%), specific phobia (43\%), ADHD (43\%), and generalized anxiety disorder (GAD) (24\%). Major depressive disorder (MDD) was found in 3/21 (14\%) of the patients. There were no significant differences with regard to sex or age for any diagnosis. More than one diagnosis was present in $33 \%$ of the sample; $24 \%$ had more than 2 diagnoses.

Phobias. Specific phobia was present in $43 \%$ of patients and was the most common type of anxiety disorder, present in 9 of the 10 subjects with an anxiety diagnosis. The most common types of specific phobia were animal, thunderstorm/lightning, and loud noises. Seven of the 9 patients had multiple phobias. No subject had social phobia.

$A D H D$. Of the subjects with ADHD, 8 of 9 were of the inattentive type, 1 was the combined type, and none were of the hyperactive-impulsive subtype.

ADIS diagnosis requires a functional interference score greater than 4 out of 8 . Many more of these subjects met a symptom-criteria for diagnosis but did not meet the requirement for functional interference. Fifteen subjects (71\%) demonstrated symptoms of specific phobia but only 9 (43\%) had significant impairment in 
functioning. Two additional subjects met criteria for GAD, and 4 additional subjects met criteria for ADHD but again did not meet the functional interference requirement.

Table I. Diagnoses in Williams Syndrome Patients

\begin{tabular}{|lll|}
\hline Diagnosis & $n$ & $\%$ \\
\hline Any Diagnosis & 13 & 62 \\
Any Anxiety Disorder & 10 & 48 \\
Separation Anxiety & 1 & 5 \\
Social Phobia & 0 & 0 \\
Specific Phobia & 9 & 43 \\
Panic Disorder & 1 & 5 \\
Agoraphobia & 1 & 5 \\
Generalized Anxiety Disorder & 5 & 24 \\
Obsessive-Compulsive Disorder & 0 & 0 \\
Post Traumatic Stress Disorder & 1 & 5 \\
Dysthymia & 2 & 10 \\
Major Depressive Disorder & 3 & 14 \\
Any ADHD & 0 & 43 \\
ADHD-Inattentive & 9 & 38 \\
ADHD-Impulsive & 1 & 0 \\
ADHD-Combined & 0 & 5 \\
Oppositional Defiant Disorder & 3 & 5 \\
\hline
\end{tabular}

\section{Family History of Psychiatric Diagnoses}

Seventy-six percent of subjects had first-degree relatives with any diagnosis on the FHS. The ADIS diagnoses used in statistical analysis were: MDD, any 
anxiety, specific phobia, and ADHD. Variables examined were: presence or absence of a diagnosis in the subject, and presence or absence of the diagnosis in first-degree relatives.

Of the WS subjects with MDD, 2 of 3 had a first-degree family history of MDD; of those without MDD, 6/18 had a first-degree family history of MDD. There was no statistically significant association between family history of MDD and subject $\operatorname{MDD}(\mathrm{p}=0.5308)$.

Of the WS subjects with any anxiety diagnosis, $4 / 10$ had a first-degree family history of anxiety; of subjects with no anxiety diagnosis, 6/11 had a first-degree family history of anxiety. There was no statistically significant association between family history of anxiety and subject anxiety $(\mathrm{p}=0.6699)$.

Of the WS subjects with a mood or anxiety disorder, 6/11 had a first-degree family history of a mood or anxiety disorder; 7/10 subjects without a mood or anxiety disorder had a first-degree family history of a mood or anxiety disorder. There was no statistically significant association between family history of mood or anxiety disorders and subject mood or anxiety disorders ( $\mathrm{p}=0.6594)$.

No WS subject with specific phobia had a family history of specific phobia (0/9). Two subjects out of 12 with no diagnosis of phobia had a family history of specific phobia. There was no statistically significant association between first-degree family history of specific phobia and subject specific phobia (p-value $=0.4857$ ).

Of the subjects with ADHD, 2/9 had a first-degree family history of ADHD; 1/12 without ADHD had a first-degree family history of ADHD. There was no statistically significant association between family ADHD and subject ADHD (pvalue $=0.5534$ ) .

In summary, no statistically significant association was found between family history of MDD, any anxiety, specific phobia, and ADHD and the presence of these disorders in WS subjects.

\section{DISCUSSION}

The WS patients of this report have a higher prevalence of psychiatric disorders compared with other populations of MR children and adults (62\% vs. 39\%) (6), as well as compared with cognitively normal children (62\% vs. 12\%) (14). An increased prevalence of psychiatric diagnoses was observed even when impairment in functioning was required for diagnosis. The rates for specific diagnoses would have been higher if not for this requirement (e.g., $71 \%$ vs. $43 \%$ for specific phobia). The use of impairment in functioning is a distinctive aspect of this study and rarely has been used in previous studies. 
Anxiety was the most prevalent diagnosis in the study population: $10 / 21$ patients (48\%). Five of these patients (24\%) had GAD while 9 of 10 (43\%) met diagnostic criteria for specific phobia. Types of phobias included animals, thunderstorms, loud noises, and high places. While some phobias may be attributed to the hyperacusis described in WS, not all phobias were related to sound. Most patients with phobias to loud noises also had phobias that were not sound-related. Despite the frequent occurrence of phobias, no patient had social phobia, consistent with the hypersociability that characterizes WS. This strong desire for social interaction appears to be an exception to the other anxieties observed in WS patients.

Previous studies of anxiety in WS have yielded similar results. Dykens reported excessive worry in 57\% of children and adults with WS (10). However, using a DSM-III-R parent interview, only $16 \%$ of patients met diagnostic criteria for GAD, similar to the $24 \%$ observed in this study. Similarly, while $96 \%$ of patients reported persistent anxiety-producing fears, only 35\% met diagnostic criteria for specific phobia (10). A multisystem study of WS adults that used the ADIS reported anxiety disorders in $65 \%$ of patients, with specific phobia being the most common subtype of anxiety (15). The present study further supports that anxiety disorders, especially specific phobia, appear to be distinctive behavioral findings in patients with WS. Of interest, despite frequent preoccupations in patients with WS, no patient met DSM-IV criteria for obsessive-compulsive disorder.

Nine patients (43\%) met criteria for ADHD, with 8 of the 9 having the inattentive type. This is higher than rates reported in studies of other MR populations (4-21\%) (1,13). Features of inattention and distractibility have previously been described in WS (16). Distinguishing ADHD from other features of mental retardation is, admittedly, difficult. For example, it may be that the hyperacusis in WS or some other attribute of MR contributes to distractibility. Alternatively, it may be that inattention is part of the behavioral phenotype of WS.

Only three patients (14\%) were found to have a history of MDD. This appears consistent with estimates in the normal pediatric population of 8-20\% (17) and in other studies of MR children of $4-10 \%$ (1).

Previous studies have not assessed the prevalence of psychiatric disorders in relatives of WS patients. Although psychiatric illness was common in first-degree relatives of the patients of this study (76\% with any diagnosis), comparison of specific diagnoses between patients and relatives failed to yield a statistically significant association for any diagnosis. The data were analyzed to detect any relationship between the presence or absence of specific psychiatric disorders in family members and the presence or absence of each disorder in the WS patient. The absence of any significant relationship with family history is consistent with

Jefferson Journal of Psychiatry, Volume 20, Number 1

ISSN 1935-0783

(C) 2006 by the authors

On the Web: jdc.jefferson.edu/jeffjpsychiatry 
our hypothesis that the presence of psychiatric illness in WS may be caused by the specific chromosomal deletion rather than the genetic contribution implicit in the family history of psychiatric illness. However, the statistical analyses performed were limited due to the small frequencies of the outcome measurements. Extrapolation of the data from the Fisher's exact test indicates that at least 60 subjects would be needed to find a statistically significant relationship among the variables. Therefore, while the results are suggestive that there may be a chromosomal link between psychiatric illness and WS, the data are insufficient to prove this conclusion.

Additional study limitations should be mentioned. As in any clinical research, it is important to acknowledge the possibility of non-response bias, specifically in this study that the families who agreed to participate may have had a different incidence of psychiatric disorders compared with those who declined. Additionally, there is an inherent recall bias in that families may under or overreport psychiatric illness in their family members in the presence of the interviewer. Findings could be enhanced in future studies by using a comparison group in the study itself, such as other MR populations. This would have been valuable because this study included the investigation of family history of psychiatric illness as well as the use of strict DSM-IV criteria in subjects, features not extensively investigated previously and therefore difficult to use in comparison analysis.

An important implication of this study is that greater attention needs to be devoted to recognizing psychiatric problems in individuals with MR, especially those with WS. Clinicians need to recognize the increased prevalence of specific disorders and to monitor for these illnesses as part of routine medical care, especially GAD, specific phobia, and ADHD. The psychiatric findings in patients with WS may provide insight into the genetic determinants of behavior and psychiatric illness in more generalized populations; therefore, additional research should continue to further investigate these relationships. Future research is also needed to systematically evaluate different treatments for anxiety disorders and ADHD in WS and other MR populations.

\section{SOURCE INFORMATION}

From the Massachusetts General Hospital, Boston, MA (J.C,K.) and the McLean Hospital, Belmont, MA (J.C.K.), Adult Psychiatry Residency Programs; the Division of Child and Adolescent Psychiatry, Department of Psychiatry, SUNY Buffalo, NY (D.L.K.) and Women and Children's Hospital of Buffalo, NY (D.L.K.); and the Division of Genetics, Department of Pediatrics, SUNY Buffalo, NY (L.S.S.) and The Williams Syndrome Clinic of Women and Children's Hospital of Buffalo, NY (L.S.S.). The authors thank the study participants and their families who gave so 
generously of their time in order to further the understanding of Williams Syndrome, and thank Tulay Koru-Sengul for her statistical assistance. Direct inquiries to Dr. Kennedy: jckennedy@partners.org

\section{REFERENCES}

1. Dykens EM. Psychopathology in children with intellectual disability. Child Psychol Psychiatry 2000; 41: 407-17

2. Singh NN, Sood A, Sonenklar N, Ellis CR. Assessment and diagnosis of mental illness in persons with mental retardation. Behav Mod 1991; 15:419-43

3. American Psychiatric Association (2000). Diagnostic and Statistical Manual of Mental Disorders, Fourth Edition, Text Revision. Washington, DC: American Psychiatric Publishing Inc.

4. Einfeld SL, Tonge BJ. Population prevalence of behavioural and emotional disturbance in children and adolescents with mental retardation. 2. Epidemiological findings. J Intellect Disabil Res 1996; 40:99-109

5. Stromme P, Diseth TH. Prevalence of psychiatric diagnoses in children with mental retardation: data from a population-based study. Dev Med Child Neurol 2000; 42: 266-70

6. Dekker MC, Koot HM. DSM-IV disorders in children with borderline to moderate intellectual disability. I: prevalence and impact. J Am Acad Child Adolesc Psychiatry 2003; 42:915-22

7. Mervis CB. Williams Syndrome: 15 years of psychological research. Dev Neuropsychol 2003; 23:1-12

8. Lashkari A, Smith AK, Graham JM, Jr. Williams-Beuren syndrome: an update and review for the primary physician Clin Pediatr 1999; 38:189-208

9. Udwin O, Yule W, Martin N. Cognitive abilities and behavioural characteristics of children with idiopathic infantile hypercalcaemia. Child Psychol Psychiatry 1987; 28:297-309

10. Dykens EM. Anxiety, fears, and phobias in persons with Williams syndrome. Dev Neuropsychol 2003; 23:291-316 
11. Silverman WK, Albano AM (1996). Anxiety Disorders Interview Schedule for DSM-IV, Child and Parent Versions. San Antonio, TX: Psychological Corporation

12. Weissman MM, Wickramaratne P, Adams P, Wolk S, Verdeli H, Olfson M. Brief screening for family psychiatric history. Arch Gen Psychiatry 2000; 57:67582

13. Szymanski L, King BH. Practice parameters for the assessment and treatment of children, adolescents, and adults with mental retardation and comorbid mental disorders. American Academy of Child and Adolescent Psychiatry Working Group on Quality Issues. J Am Acad Child Adolesc Psychiatry 1999; 38 (12 Suppl):5S$31 \mathrm{~S}$

14. Costello EJ, Egger H, Angold AJ. Ten-year research update review: the epidemiology of child and adolescent psychiatric disorders: I. methods and public health burden. J Am Acad Child Adolesc Psychiatry 2005; 44:972-86

15. Cherniske EM, Carpenter TO, Klaiman C, Young E, Bregman J, Insogna K, Schultz RT, Pober BR. Multisystem study of 20 older adults with Williams syndrome. Am J Med Genet 2004; 131:255-64

16. Greer MK, Brown FR 3rd, Pai GS, Choudry SH, Klein AJ. Cognitive, adaptive, and behavioral characteristics of Williams syndrome. Am J Med Genet 1997; 74:521-5

17. Satcher DS. Executive summary: a report of the surgeon general on mental health. Pub Health Rep 2000; 115:89-101 